\title{
Demographic Correlates of Intimate Partner Violence in the Rio San Juan Department in Nicaragua
}

\author{
Abraham. P. Buunk ${ }^{1} \&$ Pieternel Dijkstra ${ }^{2}$ \\ ${ }^{1}$ University of Curaçao, Curaçao \& University of Groningen, the Netherlands. \\ ${ }^{2}$ Private Practice for Social Psychology, Schildwolde, the Netherlands. \\ Correspondence: Abraham P. Buunk, Rozenstraat 74a, 1016 NX, Amsterdam, the Netherlands.
}

Received: June 7, 2016

doi:10.11114/ijsss.v4i8.1722

\author{
Accepted: June 21, $2016 \quad$ Available online: July 7, 2016 \\ URL: http://dx.doi.org/10.11114/ijsss.v4i8.1722
}

\begin{abstract}
Given the strong involvement of policy makers in Nicaragua with the prevention of intimate partner violence, we examined the demographic correlates of intimate partner violence in the Department of the Rio San Juan in Nicaragua, using an adapted version of the Conflict Tactics Scale. The sample consisted of 199 men and 201 women. Results showed that $35 \%$ of men and $33 \%$ of women had committed at least one act of violence. More men than women had committed serious forms violence on their partner. Overall, intimate partner violence occurred more often in urban areas than in rural areas, and most among single people, followed by cohabiting people, and next by married people. Men with a low or medium income showed of all groups the highest levels of intimate partner violence. Participants with the lowest educational level reported the highest occurrence of intimate partner violence. Results are line with those found in previous studies. Implications for policies are discussed within the article.
\end{abstract}

Keywords: intimate partner violence, physical abuse, Nicaragua, demographic factors, sex differences

\section{Introduction}

\subsection{Intimate Partner Violence}

Physical violence in intimate relationships is not a rare occurrence. On the basis of numerous studies it seems that, despite a variety in methods and in populations studied, in general somewhere around $30 \%$ of people have ever experienced some form of physical violence during their life. More specifically, in one of the first national surveys on marital violence, Murray Straus (1978) found that $28 \%$ of the American couples reported that marital violence had occurred at some point in their marriage, and that in $16 \%$ some kind of violence had occurred in the year the study was conducted. However, the most serious forms of violence, such as beating up the spouse and threatening with, or actually using, a knife or gun were very rare. In an extensive study among more than 24,000 women aged 15 to 49 from rural areas and cities in 10 countries from Asia, South America, Africa, Europe and Oceania, the reported lifetime prevalence of physical violence from one's partner varied between $22 \%$ and $45 \%$, with a mean of $36 \%$ (Garcia et al., 2006; for similar figures see Alhabib, Nur \& Jones, 2009). As in Straus's study, the ever prevalence figures for severe violence were lower, with most figures being less than $25 \%$.

Intimate partner violence poses a serious problem to both the individuals involved as well as society: it lowers the victim's quality of life, and may expose children to acts of violence, which may lead to childhood trauma (Evans, Davies \& DiLillo, 2008). In economic terms, intimate partner violence leads to increased societal costs caused by, among other things, the increased use of health services and the legal and justice system and a decrease in worker productivity (Logan, Walker, \& Hoyt, 2012). A review that converted findings for different countries into 2001 U.S. dollars (Waters et al., 2004) found the annual costs of intimate partner violence to range from $\$ 717,000$ for New Zealand to $\$ 12,6$ billion for the United States. To decrease these costs governments tend to develop policies to reduce intimate partner violence. Often, however, these policies are far from optimally effective and efficient, and some may even cause harm to victims and provide incentives for false claims (see for instance Foster, 2011). To improve these governmental policies it is of vital importance to identify populations at risk. The present study aims to contribute to this goal by studying the incidence of intimate partner violence in relation to demographic variables in the Department of the Rio San Juan in Nicaragua. More specifically, the present study examined the relations of intimate partner violence with gender, rural versus urban residence, income level, educational level, and civil status. 


\subsection{Sex Differences in Intimate Partner Violence}

It is often assumed that women, more frequently than men, are the victim of intimate partner violence. Since the controversial article by Suzanne Steinmetz (1980) who, reviewing a series of investigations, argued that husbands were the victims of marital violence as well, there has been a heavy debate on this issue. Steinmetz's data clearly indicated that nearly as many women have ever engaged in marital violence as men. In fact, in the study by Straus (1978) more women than men reported to have engaged in throwing something, kicking, biting or hitting with a fist, hitting with something, and threatening with a gun. Subsequent research using the scales developed by Straus has shown similar findings, posing a puzzle to those working with victims of physical abuse that are nearly always women. A plausible explanation is that, although both men and women engage in interpersonal partner violence, men's violent behaviors are usually more destructive, with men dominating in lethal and near-lethal violence against their partner (e.g., Archer, 2000), causing a relatively high proportion of abused women to seek help and medical attention (Langhinrichsen-Rohling, McCullars, \& Misra, 2012).

\subsection{Intimate Partner Violence and Socio-Economic Level}

There is considerable evidence that male-female intimate partner violence is more frequent among individuals of lower socio-economic levels. Figures from the Department of Justice in the United States, for instance, show that the rate of intimate violence against women generally decreases as household income levels increase (Greenfeld et al., 1998). This phenomenon is usually attributed to the fact that individuals in the lower socio-economic strata suffer from more social stress due to a higher frequency of negative life events, more stressful jobs and higher poverty (e.g., Cunradi, Cataeno \& Shafer, 2002). This social stress may translate into partner violence, also because individuals from lower socio-economic levels have fewer resources available to them to cope with stress. To date, only a few studies have explicitly investigated the relationship between partner violence committed by both men and women (i.e., mutual partner violence), and indicators of socioeconomic status. These studies seem to point in a similar direction as studies on male to female violence. In a sample of more than 20,000 US citizens, Carol Cunradi (2007), for instance, found that, as education level dropped, prevalence ratings of mutual partner violence increased. Whereas $5.3 \%$ of men with less than high school education reported mutual partner violence, $3.1 \%$ of men with a high school diploma and $2.3 \%$ of higher educated men did. Among women $5.9 \%$ with less than high school education reported mutual partner violence, whereas $4.1 \%$ of women with a high school diploma and $2.1 \%$ of higher educated women did.

\subsection{Intimate Partner Violence in Urban and Rural Areas}

There is also increasing evidence that place of residence (urban versus rural) may be an important determinant of the occurrence of intimate partner violence. Research across different samples and settings mostly shows that male-female partner violence is more prevalent in rural than in urban areas (e.g., Alio et al., 2011; Gallup-Black, 2005). Illustrative is a study by Corinne Peek-Asa et al. (2011) conducted in Iowa among almost 1500 women seeking an abortion, a group of women that, in general, relatively often suffers from partner violence. Of these women, those living in isolated rural areas reported a higher frequency and a much higher severity of abuse than did their urban counterparts. Whereas for instance $61.5 \%$ of isolated rural women reported four or more events of physical violence in the past year, 39.3\% of urban women did so. In a similar vein, more than $30 \%$ of isolated rural women reported severe to very severe physical violence compared with $10 \%$ of urban women. Comparable findings have been reported among pregnant Latinas in Arizona (Mattson, \& Rodriquez, 1999). Similar patterns are observed with regard to the murder of wives and girlfriends: rural women are far more likely to be the victim of an intimate partner murder than those living in more densely populated areas (Gallup-Black, 2005). Only a few studies have explicitly investigated the relationship between location of residenceand mutual partner violence, with both men and women being the possible perpetrator. A study among more than 1600 adults showed that women living in a rural nonfarm residence reported more frequent and severe physical abuse from their partner than women living in a farm or town. When controlling for farm residence, men who engaged in farm work - suggesting a rural place of residence - experienced more severe physical abuse and controlling abuse from their partner than men who did not engage in this type of work (Murty et al., 2013). Studies from other continents than the US also show an increased risk of partner violence for women in rural areas. For example, a study among 600 women in Nigeria, showed that the lifetime prevalence of physical violence against wives was about twice as high in in rural areas than in urban one's ( $28 \%$ vs. $14 \%)$, as was the current prevalence $(28 \%$ vs. $13 \%$; Balogun, Owoaje \& Fawole, 2012). A negative association between urban residence and wife battering was also reported in a large-scale study among almost 14,000 couples in six Sub-Saharan African countries (Alio et al, 2011).

Differences in the prevalence of intimate partner violence between rural and urban areas may be attributed to several factors. First, in rural areas, gender roles may state that wife beating is an acceptable response to a wife that shows insufficient obedience or respect or that, according to the male, fails in her role of wife and mother (Gumei et al., 2012). Second, according to Adria Gallup-Black (2005), the nature of interpersonal relationships in rural communities is very 
different from that in cities, where individuals are less likely to know each other. The close-knit nature of rural life, characterized by a lack of anonymity, may prevent women from seeking help for intimate partner violence which in turn can increase the likelihood of abuse. Finally, increased alcohol consumption in rural areas often contributes to the higher prevalence of physical violence against wives, as does the lower level of education and income, the more stressful conditions rural women live in and the lack of sufficient health and intervention programs for interpersonal partner violence (Balogun et al., 2012 Peek-Asa et al., 2011).

\subsection{The Present Study}

The present study examined the relation between intimate partner violence - as reported for oneself and for one's partner - and demographic variables, i.e., gender, place of residence (rural versus urban), income level and educational level in the Department of the Rio San Juan in Nicaragua. In addition, we examined the relation between intimate partner violence and current civil status (being married, cohabiting or single). Nicaragua is the largest country in the Central American isthmus, with a tropical climate. It is the second poorest country in the Western hemisphere (after Haiti), with in 2014 an estimated average income per capita of \$1921. It has mainly an agricultural economy and the main language is Spanish, although some indigenous tribes speak their native languages. The Department of San Juan, with a size of $7473 \mathrm{~km}$, is located in the southeast, and constitutes a rather isolated part of the country that is inhabited by around 100,000 people that live in six municipalities. Although there have been a number of important studies in Nicaragua on intimate partner violence among pregnant women, that suggest a quite high prevalence of such violence (e.g., Salazar, Valladares, Ohman, \& Högberg, 2009; Salazar, Högberg, Valladares, \& Persson, 2012), as far as could be ascertained, there have been no studies in general population samples from Nicaragua.

\section{Method}

\subsection{Sample}

The sample consisted of 199 men and 201 women from the Department of the Rio San Juan in Nicaragua. The sample did not include any couples. Half of the participants (200) came from the city of San Carlos and half from the countryside. This sample size was chosen because it allows the assessment of medium effect sizes. The men in the sample were on average significantly older than the women $[t(395)=3.63, p<.001 ; M=38.38, S D=10.36$ vs. $M=$ $34.57, S D=10.58]$. Of the women $73 \%$ were Catholic, $21 \%$ Evangelic, and the other $6 \%$ belonged to another or none religious denomination. Among men the comparable percentages were $65 \%, 22 \%$ and $13 \%$. Of the women $37 \%$ was married, $12 \%$ single, divorced or widowed, and $51 \%$ was living together. The percentages among men were virtually identical, respectively $37 \%, 14 \%$, and $49 \%$. Thus, a majority of the sample was not married and about half was living together.

As presented in Table 1, among men as well as women, there was considerable variation in the level of education, with substantial numbers of respondents in all levels except that of having no education. Women were on average somewhat more highly educated than men, $t(379)=2.95, p<.01$, which was mainly due to the actual higher number of women with a higher education. However, there were more men than women with a professional education, and overall, about half of the sample had an educational level of high school or less. There was also a considerable variation in the level of income, with substantial numbers of respondents represented in all six levels. As currently 1000 Nicaraguan Cordoba's are equivalent to US\$40, Table 1 shows that a large majority of about three quarters of the sample earned less than US $\$ 200$ a month. The difference between men and women was not significant, $t(397)=1.10, n s$.

Although not a completely a-select sample, the sample represented all existing occupational sectors in the area. As shown in Table 1, among men the largest single group worked in the agricultural sector, followed by commerce and business (mainly shopkeepers and merchants), and artisans (mainly carpenters and bricklayers). About half of the men had a job in one of these three sectors. Next, education and professional occupations (including for example accountants, statisticians and ICT specialists) accounted for another quarter of the jobs. As might be expected, the occupational pattern among women was quite different, with about a fifth of women being housewives, and a quarter being teachers, with small groups being employed in the other sectors, with commerce and business being the most important. The difference in occupational sector between men and women was significant, $\operatorname{Chi}^{2}(12)=131.29, p<.001$. 
Table 1. Educational level, income level and occupational sector for men and women separately

\begin{tabular}{|c|c|c|}
\hline & Men & Women \\
\hline \multicolumn{3}{|l|}{ Educational level } \\
\hline No education & $7 \%$ & $2 \%$ \\
\hline Primary school & $18 \%$ & $15 \%$ \\
\hline Secondary school & $31 \%$ & $30 \%$ \\
\hline Professional education & $23 \%$ & $18 \%$ \\
\hline Higher education & $16 \%$ & $31 \%$ \\
\hline Other & $5 \%$ & $4 \%$ \\
\hline \multicolumn{3}{|l|}{ Monthly income ${ }^{1}$} \\
\hline Less than 1000 Cordobas & $8 \%$ & $14 \%$ \\
\hline Between 1000 and 2000 Cordobas & $15 \%$ & $13 \%$ \\
\hline Between 2000 and 3000 Cordobas & $17 \%$ & $14 \%$ \\
\hline Between 3000 and 4000 Cordobas & $12 \%$ & $11 \%$ \\
\hline Between 4000 and 5000 Cordobas & $22 \%$ & $21 \%$ \\
\hline More than 50000 Cordobas & $27 \%$ & $25 \%$ \\
\hline \multicolumn{3}{|l|}{ Occupational sector } \\
\hline Agriculture & $19 \%$ & $8 \%$ \\
\hline Artisan & $12 \%$ & - \\
\hline Office work & $6 \%$ & $7 \%$ \\
\hline Education & $13 \%$ & $25 \%$ \\
\hline Health care & $6 \%$ & $6 \%$ \\
\hline Commerce and business & $17 \%$ & $12 \%$ \\
\hline Security & $6 \%$ & $1 \%$ \\
\hline Bars and restaurants & $2 \%$ & $6 \%$ \\
\hline Transport & $4 \%$ & - \\
\hline Professional & $10 \%$ & $3 \%$ \\
\hline Domestic & - & $9 \%$ \\
\hline Housewives & - & $21 \%$ \\
\hline Other or none & $7 \%$ & $4 \%$ \\
\hline
\end{tabular}

Note 1. 1000 Cordobas is about US\$ 40.

\subsection{Procedure}

The fieldwork was executed by a NGO, i.e., the Fundación SanLucas (San Lucas Foundation) that has through its project Arete much experience with intimate partner violence. The foundation has a networkof over 180 men and women who assist in signalling and preventing intimate partner violence, and who know thepeople in their community well. With their assistance participants were recruited in five of the six municipalities. With the exception of the small, remote town of San Juan de Nicaragua, that has only 1307 inhabitants, all municipalities in the country side were represented. First, half of the participants were recruited from the city of San Carlos from 10 neighbourhoods that were representative of the city and the other half from 12 communities in the country side that were representative of the country side. Although complete representativeness of the sample could not be realized, care was taken to obtain a sample typical of the population by as much as possible randomly approaching individuals in the different communities. Whereas only a couple of women refused to collaborate, about 50menrefused to do so. Participants received a manually rechargeable flashlight as a sign of appreciation for participating in the study.

The interviews were conducted in a place where the other spouse was not present and could not interfere. This was accomplished in nearly all cases. In a few cases, a husband suddenly appeared when his wife was being interviewed, and feared that the interviewer was making some complaint against him. Usually the interviewer could reassure the husband. For ethical reasons, the interviewers could not maintain a blind eye for apparent problems of domestic violence. As an unintended consequence of the research, a number of womenasked the interviewer forappointmentswith the psychologists of Arete regarding the problem of violence they experienced. 


\subsection{Interviews and Measures}

Most respondents did not fill out the questionnaire, but were interviewed, because many did not have a good reading ability. The interviews lasted between 45 and 60 minutes, and included a series of questions about psychological variables potentially associated with intimate partner violence (not reported here), demographic variables, and the occurrence of intimate partner violence. The violent acts against one's partner and of one's partner towards oneself were measured with a modified and extended version of the Short Form of the Revised Conflict Tactics Scale (CTS2S; Straus \& Douglas, 2004). This scale contains originally 20 items and is a short version of the Revised Conflict Tactics Scale (CTS2) that contains 78 items (Straus, Hamby, Boney-McCoy \& Sugarman, 1996). The short form was chosen to prevent annoyance among the respondents, and because it was expected that most respondents would not be able to fill out the questions themselves, but would have to be interviewed. One behavior of CTS2S was deleted, i.e., insisting on sex when the partner did not want to or insisting on sex without using a condom, without using physical force, as the second part of this item was not appropriate for the present population. On the basis of discussions with professionals of Arete, instead of using force (like hitting, holding down, or using a weapon) to make a partner have sex was replaced by the more general behavior of using force (like hitting, holding down, or using a weapon) to make one's partner do what one wanted. Therefore, another item from the CTS2S was modified into forcing one's partner to have sex when the partner didn't want to, using physical force. In addition, a couple of items from the CTS2S were replaced by the original items from the CTS2 because they contained two rather divergent behaviors. For example, the items referring to destroying something that belonged to the partner or threatening to hit the partner were replaced by items only referring to destroying something that belonged to the partner. A number of other items from the CTS2 were also included. As a result the final scale contained 30 items.

Like all questions in the questionnaire, the questions in our version of the Conflict Tactics Scale were translated into Spanish by a professional translator, next reviewed by a Dutch collaborator living in San Carlos, and finally examined and piloted by professionals from Arete among ten individuals. Two changes in the answering format were made. First, in line with the majority of studies with the CTS, we asked for the overall prevalence of the behaviors in one's relationships (Archer, 2000). Second, instead of eight possible answers as used by Straus and Douglas (2004). five possible answers were used, i.e., 'never', 'rarely (1 to 3 times), 'on various occasions' (4 to 10 times), 'often' (11 to 20 times), and 'nearly always'. Thus, like recommended by Straus, the frequency was specified rather than just giving verbal descriptions of the behaviors. In his extensive meta-analysis, John Archer (2000) presented nine items that are typically used with the Conflict Tactics Scale, and all of these were included in the present questionnaire. The items that refer to negotiation and to psychological aggression were considered filler items, and were not analyzed further, as was the single item on using force to have sex. On the basis of the remaining 22 items (referring to 11 different behaviors), in line with Straus et al. (1996) and Straus and Douglas (2004) scales were constructed for both one's own ( $M=14.07$, $S D=5.92$,alpha $=.91)$ and for one's partner's behavior $(M=14.83, S D=7.21$,alpha $=.92)$.

\section{Results}

\subsection{Incidence of Acts of Partner Violence}

First, the incidence of the various acts of intimate partner violence engaged in by one-self were examined. In Table 2 , for all behaviors, the percentages of those who had minimally engaged in it once, and of those who had minimally once experienced it on behalf of their partner are presented for men and women separately. Overall, $35 \%$ of men and 33\% of women had committed at least one act of violence. For six behaviors, men and women reported equal frequencies of violence. However, five - quite serious - forms of violence were reported significantly more by men than by women, i.e., twisting the partner's arm or hair, choking the partner, beating up the partner, beating up the partner so she or he passed out, and engaging in a fight with the partner due which she or he needed to see a doctor, but actually did not. On the total scale, men $(M=14.85, S D=6.96)$ reported having engaged more often in intimate partner violence than women $(M=13.28, S D=4.57), F(1,399)=7.00, p<.01$.

The reports on the violence by the partner showed a different picture. Overall, men and women report equal frequencies of violence by their partner; $35 \%$ of men and $36 \%$ of women said that their partner had committed at least one act of violence. Most sex differences were not significant, and three were only marginally significant, with, remarkably, men reporting somewhat more often a sprain bruise, or small cut because of fight with partner, being beating up by one's partner, and seeing a doctor because of fight with one's partner. In line with this, men indicated more often than women that they had passed out because of being beaten up by their partner. This finding suggests that many men may have tried to justify their violent behaviors by saying that their wife did the same. In line with this, the correlation between the total scales for intimate partner violence reported by oneself, and attributed to one's partner was for men $(r=.91)$ significantly higher than for women $(r=.81), z=3.97, p<.001$. In line with this, on the total scale, there was no 
significant effect of gender, $F(1,399)=1.15, p=.28$. Men $(M=15.22, S D=7.78)$ reported as much violence by their partner as women $\operatorname{did}(M=14.45, S D=6.58)$.

Table 2. Occurrence of various acts of partner violence by oneself and one's partner, for men and women separately.

\begin{tabular}{|c|c|c|c|c|c|c|}
\hline & \multicolumn{3}{|c|}{ Self } & \multicolumn{3}{|c|}{ Partner } \\
\hline & Men & Women & Significance & Men & Women & Significance \\
\hline Pushed, shoved or slapped partner & $19 \%$ & $11 \%$ & $n s$ & $25 \%$ & $29 \%$ & ns \\
\hline Destroyed something belonging to partner & $11 \%$ & $15 \%$ & $n s$ & $29 \%$ & $22 \%$ & $n s$ \\
\hline \multicolumn{7}{|l|}{ Use of force (like hitting, holding down } \\
\hline \multicolumn{7}{|l|}{ using objects, or a weapon) to have } \\
\hline partner do what she/he wanted & $15 \%$ & $10 \%$ & $n s$ & $15 \%$ & $18 \%$ & ns \\
\hline Threw something at partner that could hurt & $20 \%$ & $16 \%$ & $n s$ & $14 \%$ & $17 \%$ & ns \\
\hline Twisted partner's arm or hair & $19 \%$ & $11 \%$ & .05 & $16 \%$ & $19 \%$ & ns \\
\hline Choked partner & $18 \%$ & $9 \%$ & .001 & $18 \%$ & $14 \%$ & ns \\
\hline Beat up & $16 \%$ & $5 \%$ & .001 & $17 \%$ & $11 \%$ & .10 \\
\hline \multicolumn{7}{|l|}{ Sprain, bruise, or small cut because of } \\
\hline fight with partner & $25 \%$ & $18 \%$ & ns & $28 \%$ & $21 \%$ & .10 \\
\hline Went to see doctor because of fight & $15 \%$ & $8 \%$ & $n s$ & $16 \%$ & $12 \%$ & .10 \\
\hline \multicolumn{7}{|l|}{ with partner } \\
\hline Passed out from being beaten up & $14 \%$ & $3 \%$ & .05 & $15 \%$ & $8 \%$ & .001 \\
\hline \multicolumn{7}{|l|}{ Needed to see doctor because of fight with } \\
\hline partner, but didn't & $15 \%$ & $6 \%$ & .05 & $13 \%$ & $10 \%$ & $n s$ \\
\hline
\end{tabular}

Note. Significance refers to significance of the difference in the mean frequency of the behavior according to $t$-tests.

Similar results were obtained when using $\mathrm{Chi}^{2}$. For the last three items, self refers here to inflicting this on one's partner, partner refers here to one's partner inflicting this on oneself.

\subsection{Effects of Demographic Variables}

For each of the other demographic variables the relationship with the occurrence of intimate partner violence as reported for oneself and for one's partner was examined with a multivariate General Linear Model(GLM) with violence of oneself and of one's partner as dependent variables. Given the importance of gender differences, the main effect of gender and the interaction with the specific demographic variable were included in each analysis as well.

\subsubsection{Educational Level}

Educational level was recoded into three levels: low (primary school or less), medium (secondary school or professional education), and high (higher education). Individuals with another type of education (5\%) were excluded from the analyses. For violence by oneself there was a main effect of educational level, $F(2,380)=6.02, p<.01$, but no interaction with gender, $F(2,380)=.30, p=.74$. As Table 3 shows, overall, and among women as well as men, violence was more prevalent in the low educational level group than in both the medium and the high educational level group, whereas the medium and high educational level groups did not differ.

The same effect was obtained for violence by one's partner, i.e., a main effect of educational level, $F(2,380)=5.53, p$ $<.01$, and no interaction with gender, $F(2,380)=.05, p=.95$. As Table 3 shows, overall, and among women as well as men, violence from one's partner was more prevalent in the low educational level group than in both the medium and the high educational level group, and the medium and high educational level groups did not differ. 
Table 3. Means and standard deviations (between parentheses) for the effects of the demographic variables on intimate partner violence

\begin{tabular}{|c|c|c|c|c|c|c|c|}
\hline & & Self & & Partner & \multirow{2}{*}{\multicolumn{2}{|c|}{ Women }} & \\
\hline & Men & Women & Total & Men & & & Total \\
\hline \multicolumn{8}{|l|}{ Educational level } \\
\hline Low & $17.34^{1}(8.60)$ & $15.06^{1,2}(7.89)$ & $16.37^{1,2}(8.33)$ & $17.62^{1}(8.94)$ & \multicolumn{2}{|c|}{$16.97^{1,2}(9.60)$} & $17.34^{1,2}(9.17)$ \\
\hline Medium & $14.30^{1}(6.16)$ & $13.18^{1}(3.84)$ & $13.77^{1}(5.21)$ & $14.81^{1}(7.69)$ & \multicolumn{2}{|c|}{$14.32^{1}(6.42)$} & $14.58^{1}(7.11)$ \\
\hline High & $14.25(7.29)$ & $12.52^{2}(2.74)$ & $13.11^{2}(4.83)$ & $14.34(6.70)$ & \multicolumn{2}{|c|}{$13.26^{1,2}(4.30)$} & $13.63^{1,2}(5.36)$ \\
\hline \multicolumn{8}{|l|}{ Income } \\
\hline Low & $16.52^{1}(8.46)$ & $12.87^{1}(5.01)$ & $14.49^{1}(6.97)$ & $17.43(9.54)^{1}$ & \multicolumn{2}{|c|}{$13.64(5.92)$} & $15.32^{+}(7.93)$ \\
\hline Medium & $17.43^{1}(8.26)$ & $13.94^{1}(5.68)$ & $15.75^{2}(7.32)$ & $17.34(9.06)^{2}$ & \multicolumn{2}{|c|}{$15.19(7.58)$} & $16.31^{1}(8.41)$ \\
\hline High & $12.66(4.28)$ & $13.18(3.50)$ & $12.92^{1,2}(3.91)$ & $12.99(5.18)^{1,2}$ & \multicolumn{2}{|c|}{$14.51(6.37)$} & $13.73^{1,+}(5.83)$ \\
\hline \multicolumn{8}{|l|}{ Urban vs. Rural } \\
\hline Urban & $16.46^{1}(7.97)$ & $14.09^{1}(5.55)$ & $15.28^{1}(6.95)$ & $17.04^{1}(8.99)$ & \multicolumn{2}{|c|}{$15.17(7.41)$} & $16.11(8.22)$ \\
\hline Rural & $13.25^{1}(5.36)$ & $12.52^{1}(3.19)$ & $12.88^{1}(4.41)$ & $13.42^{1}(5.88)$ & \multicolumn{2}{|c|}{$13.75(5.74)$} & $13.58(5.80)$ \\
\hline \multicolumn{8}{|l|}{ Civil status } \\
\hline Married & $13.11^{1,2}(5.53)$ & $13.15(4.59)$ & $13.13^{1}(5.06)$ & $13.11^{1,2}(5.70)$ & 13.77 & $(6.20)$ & $13.44^{1,2}(5.95)$ \\
\hline Single $^{1}$ & $17.25^{1}(9.06)$ & $14.67(7.38)$ & $16.06^{1,2}(8.35)$ & $18.32^{1}(10.66)$ & 15.86 & $(8.69)$ & $17.19^{1}(9.79)$ \\
\hline Cohabiting & $15.48^{2}(7.00)$ & $13.08(3.60)$ & $14.25^{2}(5.64)$ & $15.94^{2}(7.84)$ & 14.61 & (6.29) & $15.26^{2}(7.10)$ \\
\hline
\end{tabular}

Note. For both dependent variables separately means in the same row with the same superscript differ significantly from each other. A + implies a marginally significant difference. In the columns for the total means for each demographic variable, means in the same column with the same superscript differ significantly from each other. All means are the original unadjusted means. ${ }^{1}$ Including widowed and divorced.

\subsubsection{Income}

Income was recoded into three levels: low (less than 2000 Cordoba's), medium (between 2000 and 4000 Cordoba's), and high (more than 4000 Cordoba's). For violence by oneself, there was a main effect of income, $\mathrm{F}(2,398)=8.82$, $\mathrm{p}$ $<.001$, and a significant interaction between gender and income, $\mathrm{F}(2,398)=6.38, \mathrm{p}<.01$. As Table 3 shows, overall, and among women as well as men, violence was more prevalent in the medium and low income groups than in the high income group, whereas the medium and low income groups did not differ. This effect was particularly pronounced among men. Contrast analyses showed that, while in the high income group men and women did not differ, $\mathrm{p}=.30$, men in both the low and medium income group showed higher levels of violence than women did, p's <.05.

For violence by one's partner, there was again a main effect of income, $F(2,398)=4.95, \mathrm{p}<.01$, as well as a significant interaction between gender and income, $\mathrm{F}(2,398)=5.27, \mathrm{p}<.01$. As shown in Table 3, violence was significantly more prevalent in the medium than in the high income group, the difference between the low and high income group was only marginally significant, and the difference between the low and medium income group was not significant. However, as shown in Table 3, the interaction implied that there was only an effect of income among men, and not among women, with men in the low income group reporting more violence from their partner than both other income groups. Additional contrast analyses showed that, while in the high income group men and women only differed marginally, $\mathrm{p}=.07$, and men and women in the medium income group did not differ, $\mathrm{p}=.19$, men in the low income group reported significantly higher levels of violence by their partner than women did, $p<.05$. Remarkably, this pattern was in part similar to the reports men gave of their own behavior.

\subsubsection{Urban versus Rural Residence}

For violence by oneself there was a main effect of urban versus rural residence, $F(2,399)=17.28, p<.001$, but no interaction with gender, $\mathrm{F}(1,399)=2.04, \mathrm{p}=.15$. As Table 3 shows, both men and women from urban neighborhoods reported more violence than people from rural communities. For violence by one's partner there was also a main effect 
of urban versus rural residence, $\mathrm{F}(2,399)=12.67, \mathrm{p}<.001$, and no interaction with gender, $\mathrm{F}(1,399)=2.39, \mathrm{p}=.12$. As Table 3 shows, people from urban neighborhoods reported more violence by their partner than people from rural communities. Although the interaction between urban versus rural residence and gender was not significant, this effect was only found among men.

\subsubsection{Civil Status}

Civil status was coded as a factor with three levels, i.e., married, cohabiting or single (including divorced and widowed). For violence by oneself there was a main effect of civil status, $F(2,399)=4.83, p<.01$, but no interaction with gender, $\mathrm{F}(2,399)=2.14, \mathrm{p}=.12$. As Table 3 shows, single people reported the most violence, significantly more than both married and cohabiting people, but the latter two groups did not differ significantly. For violence by one's partner there was also a main effect of civil status, $\mathrm{F}(2,399)=5.86, \mathrm{p}<.01$, but no interaction with gender, $\mathrm{F}(2,399)=1.27, \mathrm{p}$ $=.28$. As Table 3 shows, single people reported the most violence by their partner, significantly more than both married and cohabiting people, but, unlike what was the case for violence by oneself, married people reported less violence than cohabiting people. Although the interaction between civil status and gender was not significant, as Table 3 shows, only among men the effects of civil status were significant. To summarize, violence committed by oneself as well as by one's partner occurred most often among single people, and especially violence committed by one's partner occurred least often among married people.

To establish the independence of the effects, an overall analysis was done, including all demographic variables (gender, educational level, income, urban versus rural residence and civil status) as independent variables. This analysis showed that all variables had an independent effect on violence by oneself, all $\mathrm{F}^{\prime} \mathrm{s}>4.60$, p's $<.01$. Educational level, urban versus rural residence and civil status had also independent effects on violence by one's partner, F's $>4.36$, p's $<.05$, but this was not true for income and gender (that also did not have an effect when considered as sole independent variable), $\mathrm{F}^{\prime} \mathrm{s}<1.70$, p's $>.18$. Thus, with one exception, all main effects appeared to be independent.

\section{Discussion}

This is one of the few studies outside Western democracies on intimate partner violence that used the scales developed by Straus and his colleagues (e.g., Straus et al., 1996; Straus \& Douglas, 2004), assessing self-reports as well as partner reports of minor and serious violence. In Nicaragua, studies on intimate partner violence have been largely confined to samples of pregnant women (e.g., Salazar et al., 2009; 2012). Although the present sample was not a real a-select sample, and though the figures from various studies cannot always be compared directly due to different measures and different time frames, the results on the prevalence of intimate partner violence in the current study seem largely in line with that from other studies in many different parts of the world, i.e., that around $30 \%$ of the people have at some point in their life engaged in some form of minor intimate partner violence. This thus seems to be a more or less universal characteristic of human societies. Although $30 \%$ may seem a high figure, one needs to realize that in the majority of cases violence had occurred not more than once in a lifetime, and we feel it is exaggerated to speak of 'epidemic proportions', as sometimes is done with regard to intimate partner violence (e.g., Klein, Campbell, Soler, \& Ghez, 1997). This, however, does not imply that intimate partner violence is not a major problem that should not be addressed. As noted before, the costs for both the individual and society of intimate partner violence are extremely high, as well as the financial costs that are involved in the policies that aim to reduce intimate partner violence. In that context it is important to identify those groups that run the highest risk of being the victim of intimate partner violence. The general picture that emerges from the present study is that most intimate partner violence is reported by individuals characterized by a low educational level and a low or medium income level, who live in an urban area and who, at present, are single. Especially individuals from lower socioeconomic levels may experience stress, and take this out by acting violently towards their partner. Furthermore, the results on sex differences are in line with the conclusion from the review by Archer (2000) that the more severe forms of violence are clearly more prevalent among men than among women, clarifying why still many more women tend to seek help for intimate partner violence than men do.

\subsection{Comparison with Previous Research}

In part the present results are consistent with findings from previous studies on the relation between demographic variables and intimate partner violence. For instance, in general, low socio-economic status, as evident from, among other things, low educational level and low income, was found to be predictive of partner violence (e.g., Tang \& Lai, 2008). The most striking finding from the present study is that, in contrast to what most other studies on intimate partner violence have found, in urban areas more violence was reported than in rural areas. This may be due to inadequate sampling, that was not completely random. Nevertheless, if this finding is valid, a possible explanation is that while San Carlos is considered a city in the region, it has only around 50,000 inhabitants, and does have nothing of the characteristics of a big city such as New York, Bombay or even Amsterdam. It might rather be considered as a rural town, and in fact, many studies showing a higher incidence of intimate partner violence in the countryside, seem to have 
included cities comparable in size to San Juan. Compared to the rest of the Department of the Rio San Juan, the city of San Carlos therefore consis of a relatively urban area only. It is also possible that that there is less social control in San Carlos than in the small villages in the countryside, making intimate partner violence less likely to be noticed and reported to the police. Furthermore, housing in a city is more expensive than in rural areas. This may not only generate more financial stress, but may also result in more individuals per square meter of housing, a situation that enhances the risk of family violence (Feargemann, Lauritsen, Brink \& Mortensen, 2010). In their study in India, Lashmanan Jeyaseelan et al. (2007) found that intimate partner violence was most frequent in rural areas and in urban-slums, in contrast to urban non-slums. Nevertheless, future research definitely needs to examine the effect of different types and degrees of urbanization on intimate partner violence.

Another interesting finding of the present research was that most incidents of intimate partner violence were reported by singles and least by married individuals whereas cohabiting individuals fell in between these two with regard to the frequency of violent incidents. First, there may be a selection effect, as many violent marriages may have already ended in a divorce, causing relatively high levels of experienced partner violence among at least part of the single participants. The fact that intimate partner violence was lower among married individuals than among those living together may be explained by the fact that marriage poses a more serious commitment to the partner bond than living together (Charmichael, 2007). People may take the decision to marry less lightly than the decision to live together without being married, causing marriages to be potentially more stable and to know less sources of conflict.

\subsection{Strengths and Limitations}

Part of the data in the present study were collected in a rather isolated and remote part of Nicaragua, and interviewers had to overcome several obstacles to collect these data. This challenging endeavor, however, generated valuable data on intimate partner violence in the rural part of Nicaragua, and, as such, contributes to our knowledge and insight into the incidence of intimate partner violence in less well-developed areas. An important finding is that compared to married individuals, singles and partners living together without being married showed relatively high incidence of intimate partner violence. Our findings imply that previous studies that solely relied on samples of married individuals may underreport the incidence of intimate partner violence in society. The finding that in the present study singles had had most experiences with intimate partner violence is also an important finding for prevention. Interventions and policies aimed at reducing intimate partner violence usually focus on couples that suffer from intimate partner violence. This may make single individuals, who, as a perpetrator or a victim, have had most experiences with intimate partner violence a 'forgotten' group. The risk is that this group, once they enter a new intimate relationship, makes the same mistakes as in their former relationships and end up, again, in a violent relationship.

Of course, the present study also had some limitations. First, whereas many studies on intimate partner violence assess individuals' experiences with intimate partner violence during the past year or from their most recent partner (e.g., Ismayilova \& El-Bassel, 2013), the present study assessed the life time prevalence of intimate partner violence. This makes it hard to compare the present study's prevalence figures with those obtained by other studies that used different prevalence measures. Second, although the present study may be reasonably typical for the population of the Department of the Rio San Juan, it cannot be considered a completely representative sample for the population in this region. In addition, the fact that some difficulties were experienced in approaching the participants, may have affected the validity of the findings. Nonetheless, we feel the present study contributes significantly to the literature on intimate partner violence, by providing insight into those demographic variables that are related to the prevalence of intimate partner violence in a relatively understudied population.

\section{Acknowledgments}

This research was supported by a Netherlands Royal Academy of Sciences Grant. This research would have been impossible without the coordination and linguistic assistance by Ineke de Groot from the Stedenband Groningen-San Carlos. The interviews were conducted by Sugeyling Ruiz, Betsy Gonzalez, and Sonia Herrera of the Fundación San Lucas, and a number of interviews were done by the first author.

\section{References}

Alhabib, S., Nur, U., \& Jones, R. (2010). Domesticviolence against women: Systematic review of prevalence studies. Journal of Family Violence, 25, 369-382.http://dx.doi.org/10.1007/s10896-009-9298-4

Alio, A. P., Clayton, H. B., Garba, M., Mbah, A. K., Daley, E., \& Salihu, H. M. (2011). Spousal concordance in attitudes toward violence and reported physical abuse in African couples. 26, 2790-2800. http://dx.doi.org/10.1177/0886260510390951

Archer, J. (2000). Sex differences in aggression between heterosexual partners: A meta-analytic review. Psychological Bulletin, 126, 651-680. http://dx.doi.org/ 10.1037M J033-2909.I26.5.651 
Balogun, M. O., Owoaje, E. T., \& Fawole, O. I. (2012). Intimate partnerviolence in southwestern Nigeria: Are there rural-urban differences? Women \& Health, 52, 627-645.http://dx.doi.org/10.1080/03630242.2012.707171

Cunradi, C. B. (2007). Drinking level, neighborhood social disorder, and mutual intimate partner violence. Alcoholism: Clinical and Experimental Research, 31, 1012-1019. http://dx.doi.org/10.1111/j.1530-0277.2007.00382.x

Cunradi, C. B., Cataeno, R., \& Shafer, J. (2002). Socioeconomic predictors of intimate partner violence among White, Black, and Hispanic couples in the United States. Journal of Family Violence, 17, 377-389. http://dx.doi.org/0885-7482/02/1200-0377/0

Evans, S. E., Davies, C., \& DiLillo, D. (2008). Exposure to domestic violence: A meta-analysis of child and adolescent outcomes. Aggression and Violent Behavior, 13, 131-140. Http://dx.doi.org/10.1016/j.avb.2008.02.005

Foster, B. P. (2011). Norms and costs of government domestic violence policies: A critical review. Journal of Family and Economic Issues, 32, 140-151. http://dx.doi.org/10.1007/s10834-010-9211-6

Gallup-Black, A. (2005). Twenty years of rural and urban trends in family and intimate partner homicide: Doesplace matter? Interdisciplinary \& International Journal, 9, 149-173. Http://dx.doi.org/ 10.1177/1088767904274158

Garcia-Moreno, C., Ellsberg, M. Heise, \&Watts,L. C. H. (2006). Prevalence of intimate partner violence: Findings from the WHO multi-country study on women's health and domestic violence. The Lancet, 368, 1260-1269. http://dx.doi.org/10.1016/S0140-6736(06)69523-8

Greenfeld, L. A., Rand, M. R., Craven, D., Klaus, P. A., Perkins C. A., Ringel, C., Warchol, G., Maston, C., \& Fox, J. A. (1998). Violence by intimates: Analysis of data on crimes by current or former spouses, boyfriends, and girlfriends. Washington, DC: U.S. Department of Justice, Office of Justice Programs, Bureau of Justice Statistics. See: http://bjs.gov/content/pub/pdf/vi.pdf

Ismayilova, L., \& El-Bassel, N. (2013). Prevalence and correlates of intimate partnerviolence by type and severity: Population-based studies in Azerbaijan, Moldova, and Ukraine. Journal of Interpersonal Violence, 28, 2521-2556. http://dx.doi.org/ 10.1177/0886260513479026

Langhinrichsen-Rohling, J., McCullars, A., \& Misra, T. A. (2012). Motivations for men and women's intimate partner violence perpetration: A comprehensive review. Partner Abuse, 3, 429-468. http://dx.doi.org/10.1891/1946-6560.3.4.429

Klein, E., Campbell, J., Soler, E., \& Ghez, M. (1997). Ending domesticviolence: Changing public perceptions: halting the epidemic. Thousand Oaks, US: Sage Publications.

Logan, T. K., Walker, R., \& Hoyt, W. (2012). The economic costs of partner violence and the cost-benefit of civil protective orders. Journal of Interpersonal Violence, 27, 1137-1154. http://dx.doi.org/10.1177/0886260511424500

Mattson, S., \& Rodriquez, E. (1999). Battering in pregnant Latinas. Issues in Mental Health Nursing, 20, 405-422.

Murty, S. A., Peek-Asa, C., Zwerling, C., Stromquist, A., Burmeister, L. F., \& Merchant, J. A. (2003).Physical and emotional partner abuse reported by men and women in a rural community. American Journal of Public Health, 93, 1073-1075.

Peek-Asa, C., Wallis, A., Harland, K., Beyer, K., Dickey, P.B.S., \& Saftlas, A. (2011). Rural disparity in domestic violence prevalence and access to resources. Journal ofWomen's Health, 20, 1743-1749. http://dx.doi.org/10.1089/jwh.2011.2891

Salazar, M., Valladares, E., Ohman, A., \& Högberg, U. (2009). Ending intimate partner violence after pregnancy:findings from a community-based longitudinal study in Nicaragua. BMC Public Health, 9, 350.http://dx.doi.org/10.1186/1471-2458-9-350

Salazar, M., Högberg, U., Valladares, E., \& Persson, L. (2012). Intimate partner violence and early child growth: a community-based cohort study in Nicaragua. BMC Pediatrics, 12, 82. http://dx.doi.org/10.1186/1471-2431-12-82

Steinmetz, S.K. (1980). Women and violence: Victims and perpetrators. American Journal of Psychotherapy, 34, 334-350.

Straus, M. A. (1977-1978). Wife beating: How common and why? Victimology: An International Journal, 2, 443- 458.

Straus, M. A., \& Douglas, E. M. (2004). A short form of the revised conflict tactics scales, and typologies forseverity and mutuality. Violence and Victims, 19, 507-520.http://dx.doi.org/10.1891/vivi.19.5.507.63686

Straus, M. A., Hamby, S. L., Boney-McCoy, S., \& Sugarman, D. B. (1996). The revised Conflict Tactics Scales (CTS2): Development and preliminary psychometric data. Journal of Family Issues, 17, 283-316. 
Tang, C. S., \& Lai, B. P. (2008). A review of empirical literature on the prevalence and risk markers of male-on-female intimate partner violence in contemporary China, 1987-2006. Aggression and Violent Behavior, 13, 10-28. http://dx.doi.org/10.1016/j.avb.2007.06.001

Waters, R. H., Hyder, A. A., Rajkotia, Y., Basu, S., \& Butchart, A. (2005). The costs of interpersonal violence: An international review. Health Policy, 73, 303-315. http://dx.doi.org/10.1016/j.healthpol.2004.11.022

This work is licensed under a Creative Commons Attribution 3.0 License. 